\title{
A DOSY Spectroscopy Study of Proton Exchange Processes Involving Inner N-H Protons in Natural and Synthetic Porphyrins
}

\author{
Dmitriy V. Belykh ${ }^{\mathrm{a}}$ and Alexander L. Stolypko ${ }^{\mathrm{a}, \mathrm{b}}$ \\ anstitute of Chemistry, Komi Science Centre, Ural Branch of the Russian Academy of Sciences, 167982 Syktyvkar, Russia \\ ${ }^{\mathrm{b}}$ Syktyvkar State University, 167000 Syktyvkar, Russia \\ ${ }^{\circledR}$ Corresponding authorE-mail: belykh-dv@mail.ru
}

\begin{abstract}
DOSY spectroscopy was used to assess the involvement of inner $N$-H protons of porphyrinic compounds in exchange processes. The assessment was made by comparison of increase of self-diffusion coefficients of $N-H$ and $C$ - $H$ protons. The difference in the measured coefficients can serve as a quantitative measure of hydrogen exchange intensity and thus of $\mathrm{N}-\mathrm{H}$ acidity. Using DOSY allowed to demonstrate that regardless of the strongly different chemical shifts, the $N-H$ bonds in porphyrins and chlorins have similar acidity and the magnetically non-equivalent inner $N-H$ protons in chlorins have a different degree of involvement in exchange processes.
\end{abstract}

Keywords: Porphyrins, DOSY spectroscopy, exchange process.

\section{Обменные процессы с участием внутрициклических NH-протонов природных и синтетических порфиринов по Аанным DOSY}

\author{
A. В. Белых, ${ }^{a @ ~ A . ~} \lambda$. Столыпко ${ }^{a, b}$ \\ а Институт химии Коми научного центра Уральского отделения Российской академии наук, 167982 Сыктылкар, \\ Россия \\ ${ }^{\mathrm{b}}$ Сыктывкарский государственный университет, 167000 Сыктывкар, Россия \\ @E-mail: belykh-dv@mail.ru
}

Методом DOSY выполнена оценка участия внутрициклических NH-протонов порфириновых соединений в обменных процессах. Обмен был зафиксирован путем сравнения увеличения коэффициентов самодиффузии NH-протонов по сравнению с CH-протонами молекуль. Разница измеряемых коэффициентов самодиффузии NH- и CH-протонов молекулы может служить количественной мерой интенсивности межмолекулярных обменных процессов и, следовательно, мерой кислотности связи N-H. Применение DOSY позволило установить, что, несмотря на сильнье различия в химических сдвигах, кислотности связей N-H в порфиринах и хлоринах близки, а магнитно неэквивалентные внутрициклические NH-протонь хлоринов задействованы в обменных процессах в разной степени.

Ключевые слова: Порфирины, спектроскопия DOSY, обменный процесс. 


\section{Introduction}

Studies of the structure and reactivity of porphyrins present great interest because these macrocyclic compounds possess unique properties. The porphyrinic core coordination site hardly has any analogs in other compounds and the processes it participates in (complex formation, acid-base equilibria, tautomerism) are important for various areas of chemistry. ${ }^{[1-5]}$ Many reactions involving this coordination site are one or another way linked to the dissociation of $\mathrm{N}-\mathrm{H}$ bonds, so the information on the inner hydrogen atoms is important for prediction of porphyrin reactivity. The strength of the N-H bond can be evaluated by studies of different processes that result in its cleavage. Such an approach has a number of drawbacks, caused by, firstly, the difficulty to distinguish the individual influence of many experimental factors on the test reactions (complex formation, deprotonation, etc.), and secondly - interference from side reactions of the more reactive substituents of the tetrapyrrolic core. Therefore, new ways of quantitative evaluation of inner N-H protons and the whole coordination site should be developed. It would be convenient if the evaluation procedure did not require harsh conditions. Moreover, evaluation under mild conditions would give a universal measurement scale. DOSY spectroscopy allows to determine the self-diffusion coefficients and thus to assess the mobility of a molecule in solutions. The $\mathrm{N}-\mathrm{H}$ protons in porphyrins are known to take part in tautomeric transformations (the so called N-NH-tautomerism, i.e. fast hydrogen exchange between the nitrogen atoms of the $\mathrm{H}_{2} \mathrm{~N}_{4}$ coordination site) $)^{[1-5]}$ as well as in intramolecular proton exchange processes. Both can contribute to the self-diffusion coefficient (D) of N-H protons which is measured by DOSY experiments. Such contributions result in the $\mathrm{D}$ values for $\mathrm{N}-\mathrm{H}$ protons to be greater than the $\mathrm{D}$ values for $\mathrm{C}-\mathrm{H}$ protons of the rest of the molecule. The current study presents an analysis of DOSY experiments for a number of natural and synthetic porphyrins (1-11) (Scheme 1) conducted in an attempt to assess the contribution of exchange processes to the $\mathrm{D}$ coefficients of $\mathrm{N}-\mathrm{H}$ protons determined by DOSY spectroscopy.

\section{Experimental}

Octaethylporphyrin (1) (Sigma-Aldrich) was used as received. Methyl pyropheophorbide a (8), protoporphyrin dimethyl ester (2), deuteroporphyrin dimethyl ester (3) and tetra(meso)phenylporphyrin (4) were obtained according to the known procedure. ${ }^{[6]}$ Methyl pheophorbide $a(\mathbf{1})$, its 13(2)-hydroxy derivative (7) and methyl pheophorbide $b(\mathbf{9})$ were isolated from the lipid fraction of Serratula coronata extract according to ${ }^{[7]}$. Dibutylamide (6) was synthesized according to ${ }^{[8]}$. Chlorin $e_{6}$ dimethylamide (10) was obtained according to ${ }^{[9]}$. Chlorin $e_{6}$ methylamide (11) was synthesized according to $^{[10]}$. The spectral data of the compounds studied are given below. The NMR experiments were conducted on a Bruker Avance II spectrometer ( $300 \mathrm{MHz}$ ). The working concentrations were $0.01-0.02 \mathrm{mmol} / \mathrm{mL}$ in $\mathrm{CDCl}_{3}$. Water content in $\mathrm{CDCl}_{3}$ was $0.01 \mathrm{mmol} / \mathrm{mL}$. Experimental parameters: «double stimulated echo with bipolar pulsed gradients» impulse sequence was performed on a Bruker Avance II spectrometer by a standard convection compensating program dstebpgp3s, diffusion time D20 $=0.1 \mathrm{~s}$; gradient impulse time $\mathrm{P} 30=1600 \mathrm{mcs}$; number of scans NS=16; number of gradient spectra td $(\mathrm{F} 1)=32$, each with $16 \mathrm{k}$ discretization frequency.
The experiment was performed at a stabilized temperature $\left(24{ }^{\circ} \mathrm{C}\right)$ and an air cushion was used to dampen external vibrations. The thermostat airflow was $670 \mathrm{~L} / \mathrm{h}$.

The ${ }^{1} \mathrm{H}$ NMR spectral data for the studied compounds are given below $\left(\mathrm{CDCl}_{3}, 300 \mathrm{MHz}\right.$, number of scans $\mathrm{NS}=8$, discretization frequency $16 \mathrm{k}), \delta$, ppm.

2,3,7,8,12,13,17,18-Octaethylporphyrin (1): $10.15 \mathrm{~s}\left(4 \mathrm{H}, \mathrm{H}^{5}\right.$, $\mathrm{H}^{10}, \mathrm{H}^{15}$ and $\left.\mathrm{H}^{20}\right) ; 4.15 \mathrm{q}\left(16 \mathrm{H}, 7.3 \mathrm{~Hz}, 2,3,7,8,12,13,17,18-\mathrm{CH}_{2} \mathrm{CH}_{3}\right)$; $1.97 \mathrm{t}\left(24 \mathrm{H}, 7.3 \mathrm{~Hz}, 2,3,7,8,12,13,17,18-\mathrm{CH}_{2} \mathrm{CH}_{3}\right)$.

Protoporphyrin dimethyl ester (2): 10.12 s $\left(1 \mathrm{H}, \mathrm{H}^{10}\right)$, $10.10 \mathrm{~s}\left(1 \mathrm{H}, \mathrm{H}^{5}\right), 9.99 \mathrm{~s}\left(1 \mathrm{H}, \mathrm{H}^{20}\right), 9.98 \mathrm{~s}\left(1 \mathrm{H}, \mathrm{H}^{20}\right), 8.27 \mathrm{dd}[1 \mathrm{H}$, $8-\left(\mathrm{CH}=\mathrm{CH}_{2}\right), J 17.6$ and $\left.11.6 \mathrm{~Hz}\right], 8.25 \mathrm{dd}\left[1 \mathrm{H}, 3-\left(\mathrm{CH}=\mathrm{CH}_{2}\right), J 17.6\right.$ and $11.7 \mathrm{~Hz}], 6.38$ br.d [2H, 3- $\left(\mathrm{CH}=\mathrm{CH} H_{\text {trans }}\right)$ and $8-\left(\mathrm{CH}=\mathrm{CH} H_{\text {trans }}\right)$, $J 17.6 \mathrm{~Hz}$ ], 6.20 br.d [2H, $3-\left(\mathrm{CH}=\mathrm{CH} H_{\text {cis }}\right)$ and $8-\left(\mathrm{CH}=\mathrm{CH} H_{\text {cis }}\right), J$ $11.6 \mathrm{~Hz}], 4.40 \mathrm{t}\left(4 \mathrm{H}, 13-\right.$ and $\left.17-\mathrm{CH}_{2} \mathrm{CH}_{2} \mathrm{CO}_{2} \mathrm{CH}_{3}, J 8.1 \mathrm{~Hz}\right), 3.70$ $\mathrm{s}\left(6 \mathrm{H}, 13-\right.$ and $\left.17-\mathrm{CH}_{2} \mathrm{CH}_{2} \mathrm{CO}_{2} \mathrm{CH}_{3}\right), 3.69 \mathrm{~s}\left(3 \mathrm{H}, 7-\mathrm{CH}_{3}\right), 3.67 \mathrm{~s}$ $\left(3 \mathrm{H}, 12-\mathrm{CH}_{3}\right), 3.61 \mathrm{~s}\left(6 \mathrm{H}, 12-\mathrm{CH}_{3}\right.$ and $\left.18-\mathrm{CH}_{3}\right), 3.29 \mathrm{t}(4 \mathrm{H}, 13-$ and $\left.17-\mathrm{CH}_{2} \mathrm{CH}_{2} \mathrm{CO}_{2} \mathrm{CH}_{3}, J 8.1 \mathrm{~Hz}\right),-3.87$ br.s (2H, I- and III-NH).

Deuteroporphyrin dimethyl ester (3): 10.19, 10.15, 10.12 and 10.08 all s $\left(1 \mathrm{H}, \mathrm{H}^{5}, \mathrm{H}^{10}, \mathrm{H}^{15}\right.$ and $\left.\mathrm{H}^{20}\right) ; 9.15$ and 9.13 both $\mathrm{s}\left(1 \mathrm{H}, \mathrm{H}^{3}\right.$ and $\left.\mathrm{H}^{8}\right) ; 7.47 \mathrm{t}\left(4 \mathrm{H}, 13-\right.$ and $\left.17-\mathrm{CH}_{2} \mathrm{CH}_{2} \mathrm{CO}_{2} \mathrm{CH}_{3}\right) ; 3.80$ and 3.78 both s (3H, 13- and 17- $\left.\mathrm{CH}_{2} \mathrm{CH}_{2} \mathrm{CO}_{2} \mathrm{CH}_{3}\right) ; 2-, 7-$ and $11-\mathrm{CH}_{3}: 3.70 \mathrm{~s}$ $(6 \mathrm{H})$ and $3.68 \mathrm{~s}(3 \mathrm{H}) ;-3.80$ br.s (2H, I- and III-NH).

5,10,15,20-Tetraphenylporphyrin (4): $8.90 \mathrm{~s}\left(8 \mathrm{H}, \mathrm{H}^{2}, \mathrm{H}^{3}, \mathrm{H}^{7}\right.$, $\mathrm{H}^{8}, \mathrm{H}^{12}, \mathrm{H}^{13}, \mathrm{H}^{17}$, and $\left.\mathrm{H}^{18}\right), 8.30-8.23 \mathrm{~m}(8 \mathrm{H}, o-\mathrm{H}, \mathrm{Ph}), 7.84-7.77 \mathrm{~m}$ $(12 \mathrm{H}, m, p-\mathrm{H}, \mathrm{Ph}),-2.72$ br.s (2H, I, III-NH).

Methyl pheophorbide a (5): $9.56 \mathrm{~s}\left(1 \mathrm{H}, \mathrm{H}^{10}\right), 9.42 \mathrm{~s}\left(1 \mathrm{H}, \mathrm{H}^{5}\right)$, $8.60 \mathrm{~s}\left(1 \mathrm{H}, \mathrm{H}^{20}\right), 8.03 \mathrm{dd}\left[1 \mathrm{H}, 3-\left(\mathrm{CH}=\mathrm{CH}_{2}\right), J 17.6\right.$ and $\left.11.7 \mathrm{~Hz}\right]$, $6.33 \mathrm{dd}\left[1 \mathrm{H}, 3-\left(\mathrm{CH}=\mathrm{CH} H_{\text {trans }}\right), J 17.6\right.$ and $\left.1.5 \mathrm{~Hz}\right], 6.29 \mathrm{~s}\left(1 \mathrm{H}, \mathrm{H}^{13(2)}\right)$, $6.21 \mathrm{dd}\left[1 \mathrm{H}, 3-\left(\mathrm{CH}=\mathrm{CHH}_{\text {cis }}\right), J 11.7\right.$ and $\left.1.5 \mathrm{~Hz}\right], 4.50 \mathrm{qd}\left(1 \mathrm{H}, \mathrm{H}^{18}\right.$, $J 7.3$ and $2.2 \mathrm{~Hz}), 4.24$ br. dt $\left(1 \mathrm{H}, \mathrm{H}^{17}, J 8.8\right.$ and $\left.2.2 \mathrm{~Hz}\right), 3.91 \mathrm{~s}$ $\left(3 \mathrm{H}, 13(2)-\mathrm{COOCH}_{3}\right), 3.72 \mathrm{q}\left(2 \mathrm{H}, 8-\mathrm{CH}_{2} \mathrm{CH}_{3}, J 7.3 \mathrm{~Hz}\right), 3.72 \mathrm{~s}(3 \mathrm{H}$, $\left.17-\mathrm{CH}_{2} \mathrm{CH}_{2} \mathrm{COOCH}_{3}\right), 3.61 \mathrm{~s}\left(3 \mathrm{H}, 12-\mathrm{CH}_{3}\right), 3.44 \mathrm{~s}\left(3 \mathrm{H}, 2-\mathrm{CH}_{3}\right)$, $3.27 \mathrm{~s}\left(3 \mathrm{H}, 7-\mathrm{CH}_{3}\right), 2.75-2.49 \mathrm{~m}\left[2 \mathrm{H}, 17-\left(\mathrm{CH}_{2} \mathrm{CH}_{2} \mathrm{COOCH}_{3}\right)\right]$, 2.43-2.20 m [2H, 17- $\left.\left(\mathrm{CH}_{2} \mathrm{CH}_{2} \mathrm{COOCH}_{3}\right)\right], 1.85 \mathrm{~d}\left(3 \mathrm{H}, 18-\mathrm{CH}_{3}, J\right.$ $7.3 \mathrm{~Hz}), 1.73 \mathrm{t}\left(8-\mathrm{CH}_{2} \mathrm{CH}_{3}, J 7.3 \mathrm{~Hz}\right), 0.59$ br.s. $(1 \mathrm{H}, \mathrm{I}-\mathrm{NH}),-1.58$ br.s. (1H, III-NH).

Pheophorbide a 13(2)-N,N-dibutylamide 17 methyl ester (6): $9.56 \mathrm{~s}\left(1 \mathrm{H}, \mathrm{H}^{10}\right), 9.44 \mathrm{~s}\left(1 \mathrm{H}, \mathrm{H}^{5}\right), 8.69 \mathrm{~s}\left(1 \mathrm{H}, \mathrm{H}^{20}\right), 8.05 \mathrm{dd}[1 \mathrm{H}$, $3-\left(\mathrm{CH}=\mathrm{CH}_{2}\right), J 17.6$ and $\left.11.7 \mathrm{~Hz}\right], 6.68 \mathrm{~s}\left(1 \mathrm{H}, \mathrm{H}^{13(2)}\right), 6.32 \mathrm{~d}[1 \mathrm{H}$, $\left.3-\left(\mathrm{CH}=\mathrm{CH} H_{\text {trans }}\right), J 17.6 \mathrm{~Hz}\right], 6.21 \mathrm{~d}\left[1 \mathrm{H}, 3-\left(\mathrm{CH}=\mathrm{CH} H_{\text {cis }}\right), J 11.7 \mathrm{~Hz}\right]$, $4.47 \mathrm{q}\left(1 \mathrm{H}, \mathrm{H}^{18}, J 7.3 \mathrm{~Hz}\right), 4.20$ br.d $\left(1 \mathrm{H}, \mathrm{H}^{17}, J 8.1 \mathrm{~Hz}\right), 3.74 \mathrm{q}(2 \mathrm{H}$, $\left.8-\mathrm{CH}_{2} \mathrm{CH}_{3}, J 7.3 \mathrm{~Hz}\right), 3.70 \mathrm{~s}\left(3 \mathrm{H}, 17-\mathrm{CH}_{2} \mathrm{CH}_{2} \mathrm{COOCH}_{3}\right), 3.51 \mathrm{~s}$ $\left(3 \mathrm{H}, 12-\mathrm{CH}_{3}\right), 3.45 \mathrm{~s}\left(3 \mathrm{H}, 2-\mathrm{CH}_{3}\right), 3.29 \mathrm{~s}\left(3 \mathrm{H}, 7-\mathrm{CH}_{3}\right), 2.47-2.16 \mathrm{~m}$ $\left[4 \mathrm{H}, 17-\left(\mathrm{CH}_{2} \mathrm{CH}_{2} \mathrm{COOCH}_{3}\right)\right], 1.82 \mathrm{~d}\left(3 \mathrm{H}, 18-\mathrm{CH}_{3}, J 7.3 \mathrm{~Hz}\right), 1.74$ $\mathrm{t}\left(8-\mathrm{CH}_{2} \mathrm{CH}_{3}, \mathrm{~J} 7.3 \mathrm{~Hz}\right), 13(2)-\mathrm{CON}\left(\mathrm{CH}_{2} \mathrm{CH}_{2} \mathrm{CH}_{2} \mathrm{CH}_{3}\right)_{2}: 3.64-3.51$ $\mathrm{m}(2 \mathrm{H}), 2.70-2.47 \mathrm{~m}(2 \mathrm{H}), 1.94-1.64 \mathrm{~m}(3 \mathrm{H}), 1.56-1.17 \mathrm{~m}(4 \mathrm{H})$, $1.08-0.79 \mathrm{~m}(4 \mathrm{H}), 1.01 \mathrm{t}(3 \mathrm{H}, J 7.3 \mathrm{~Hz}), 0.46$ br.s (1H, I-NH), -1.64 br.s (1H, III-NH).

13(2)-Hydroxypheophorbide a methyl ester (7): $9.66 \mathrm{~s}(1 \mathrm{H}$, $\left.\mathrm{H}^{10}\right), 9.52 \mathrm{~s}\left(1 \mathrm{H}, \mathrm{H}^{5}\right), 8.68 \mathrm{~s}\left(1 \mathrm{H}, \mathrm{H}^{20}\right), 8.06 \mathrm{dd}\left[1 \mathrm{H}, 3-\left(\mathrm{CH}=\mathrm{CH}_{2}\right)\right.$, $J 17.6$ and $11.7 \mathrm{~Hz}], 6.35 \mathrm{~d}\left[1 \mathrm{H}, 3-\left(\mathrm{CH}=\mathrm{CH} H_{\text {trans }}\right), J 17.6 \mathrm{~Hz}\right]$, $6.24 \mathrm{~d}\left[1 \mathrm{H}, 3-\left(\mathrm{CH}=\mathrm{CH} H_{\text {cis }}\right), J 11.7 \mathrm{~Hz}\right], 5.51 \mathrm{~s}(1 \mathrm{H}, 13(2)-\mathrm{OH})$, $4.53 \mathrm{q}\left(1 \mathrm{H}, \mathrm{H}^{18}, J 7.4 \mathrm{~Hz}\right), 4.19$ br.d $\left(1 \mathrm{H}, \mathrm{H}^{17}, J 8.8 \mathrm{~Hz}\right), 3.84$ $3.77 \mathrm{~m}\left(2 \mathrm{H}, 8-\mathrm{CH}_{2} \mathrm{CH}_{3}\right), 3.77 \mathrm{~s}\left(3 \mathrm{H}, 13(2)-\mathrm{COOCH}_{3}\right), 3.69 \mathrm{~s}(3 \mathrm{H}$, $\left.17-\mathrm{CH}_{2} \mathrm{CH}_{2} \mathrm{COOCH}_{3}\right), 3.66 \mathrm{~s}\left(3 \mathrm{H}, 12-\mathrm{CH}_{3}\right), 3.47 \mathrm{~s}\left(3 \mathrm{H}, 2-\mathrm{CH}_{3}\right)$, $3.30 \mathrm{~s}\left(3 \mathrm{H}, 7-\mathrm{CH}_{3}\right), 17-\mathrm{CH}_{2} \mathrm{CH}_{2} \mathrm{COOCH}_{3}: 3.06-2.87 \mathrm{~m}(1 \mathrm{H})$ and $2.67-2.50 \mathrm{~m}(1 \mathrm{H}) ; 2.41-2.23 \mathrm{~m}\left[2 \mathrm{H}, 17-\left(\mathrm{CH}_{2} \mathrm{CH}_{2} \mathrm{COOCH}_{3}\right)\right]$, $1.75 \mathrm{t}\left(8-\mathrm{CH}_{2} \mathrm{CH}_{3}, J 7.3 \mathrm{~Hz}\right), 1.64 \mathrm{~d}\left(3 \mathrm{H}, 18-\mathrm{CH}_{3}, J 8.00 \mathrm{~Hz}\right)$, 0.37 br.s $(1 \mathrm{H}, \mathrm{I}-\mathrm{NH}),-1.78$ br.s $(1 \mathrm{H}, \mathrm{III}-\mathrm{NH})$.

Methyl pyropheophorbide a $(\mathbf{8}): 9.55 \mathrm{~s}\left(1 \mathrm{H}, \mathrm{H}^{10}\right), 9.43 \mathrm{~s}(1 \mathrm{H}$, $\left.\mathrm{H}^{5}\right), 8.59 \mathrm{~s}\left(1 \mathrm{H}, \mathrm{H}^{20}\right), 8.05 \mathrm{dd}\left[1 \mathrm{H}, 3-\left(\mathrm{CH}=\mathrm{CH}_{2}\right), J 17.6\right.$ and $\left.11.7 \mathrm{~Hz}\right]$, $6.32 \mathrm{~d}\left[1 \mathrm{H}, 3-\left(\mathrm{CH}=\mathrm{CH} H_{\text {trans }}\right), J 17.6 \mathrm{~Hz}\right], 6.21 \mathrm{~d}\left[1 \mathrm{H}, 3-\left(\mathrm{CH}=\mathrm{CH} H_{\text {cis }}\right)\right.$, $J 11.7 \mathrm{~Hz}], 5.31 \mathrm{~d}\left(1 \mathrm{H}, \mathrm{H}^{13(2)}, J 19.8 \mathrm{~Hz}\right), 5.15 \mathrm{~d}\left(1 \mathrm{H}, \mathrm{H}^{13(2)}{ }_{\mathrm{B}}, J\right.$ $20.1 \mathrm{~Hz}), 4.53 \mathrm{qd}\left(1 \mathrm{H}, \mathrm{H}^{18}, J 7.3\right.$ and $\left.2.2 \mathrm{~Hz}\right), 4.34 \mathrm{br}$. dt $\left(1 \mathrm{H}, \mathrm{H}^{17}\right.$, $J 8.1$ and $2.9 \mathrm{~Hz}), 3.74 \mathrm{q}\left(2 \mathrm{H}, 8-\mathrm{CH}_{2} \mathrm{CH}_{3}, J 8.1 \mathrm{~Hz}\right), 3.71 \mathrm{~s}(3 \mathrm{H}$, $\left.17-\mathrm{CH}_{2} \mathrm{CH}_{2} \mathrm{COOCH}_{3}\right), 3.65 \mathrm{~s}\left(3 \mathrm{H}, 12-\mathrm{CH}_{3}\right), 3.45 \mathrm{~s}\left(3 \mathrm{H}, 2-\mathrm{CH}_{3}\right)$, $3.28 \mathrm{~s}\left(3 \mathrm{H}, 7-\mathrm{CH}_{3}\right), 2.82-2.53 \mathrm{~m}\left[2 \mathrm{H}, 17-\left(\mathrm{CH}_{2} \mathrm{CH}_{2} \mathrm{COOCH}_{3}\right)\right]$, 
2.43-2.25 m [2H, 17- $\left.\left(\mathrm{CH}_{2} \mathrm{CH}_{2} \mathrm{COOCH}_{3}\right)\right], 1.85 \mathrm{~d}\left(3 \mathrm{H}, 18-\mathrm{CH}_{3}, \mathrm{~J}\right.$ $7.4 \mathrm{~Hz}), 1.74 \mathrm{t}\left(8-\mathrm{CH}_{2} \mathrm{CH}_{3}, J 7.3 \mathrm{~Hz}\right), 0.52$ br.s $(1 \mathrm{H}, \mathrm{I}-\mathrm{NH}),-1.64$ br.s (1H, III-NH).

Methyl pheophorbide $b(9): 11.06 \mathrm{~s} \quad(1 \mathrm{H}, 3-\mathrm{CH}=\mathrm{O})$, $10.27 \mathrm{~s}\left(1 \mathrm{H}, \mathrm{H}^{5}\right), 9.52 \mathrm{~s}\left(1 \mathrm{H}, \mathrm{H}^{10}\right), 8.57 \mathrm{~s}\left(1 \mathrm{H}, \mathrm{H}^{20}\right), 7.99 \mathrm{dd}[1 \mathrm{H}$, $3-\left(\mathrm{CH}=\mathrm{CH}_{2}\right), J 17.6$ and $\left.11.7 \mathrm{~Hz}\right], 6.39 \mathrm{~d}\left[1 \mathrm{H}, 3-\left(\mathrm{CH}=\mathrm{CH} H_{\text {trans }}\right)\right.$, $J 17.6 \mathrm{~Hz}], 6.27 \mathrm{~s}\left(1 \mathrm{H}, \mathrm{H}^{13(2)}\right), 6.25 \mathrm{~d}\left[1 \mathrm{H}, 3-\left(\mathrm{CH}=\mathrm{CH} H_{\text {cis }}\right), J 11.7\right.$ $\mathrm{Hz}], 4.50 \mathrm{qd}\left(1 \mathrm{H}, \mathrm{H}^{18}, J 7.3\right.$ and $\left.2.2 \mathrm{~Hz}\right), 4.24 \mathrm{br}$. dt $\left(1 \mathrm{H}, \mathrm{H}^{17}, J 8.1\right.$ and $2.2 \mathrm{~Hz}), 3.95 \mathrm{~s}\left(3 \mathrm{H}, 13(2)-\mathrm{COOCH}_{3}\right), 3.93 \mathrm{q}\left(2 \mathrm{H}, 8-\mathrm{CH}_{2} \mathrm{CH}_{3}\right.$, $J 8.1 \mathrm{~Hz}), 3.68 \mathrm{~s}\left(3 \mathrm{H}, 17-\mathrm{CH}_{2} \mathrm{CH}_{2} \mathrm{COOCH}_{3}\right), 3.64 \mathrm{~s}\left(3 \mathrm{H}, 12-\mathrm{CH}_{3}\right)$, $3.41 \mathrm{~s}\left(3 \mathrm{H}, 2-\mathrm{CH}_{3}\right), 2.78-2.52 \mathrm{~m}\left[2 \mathrm{H}, 17-\left(\mathrm{CH}_{2} \mathrm{CH}_{2} \mathrm{COOCH}_{3}\right)\right]$, 2.43-2.25 m [2H, 17- $\left.\left(\mathrm{CH}_{2} \mathrm{CH}_{2} \mathrm{COOCH}_{3}\right)\right], 1.88 \mathrm{~d}\left(3 \mathrm{H}, 18-\mathrm{CH}_{3}\right.$, $J 7.3 \mathrm{~Hz}), 1.79$ t $\left(8-\mathrm{CH}_{2} \mathrm{CH}_{3}, J 7.3 \mathrm{~Hz}\right), 0.49$ br.s $(1 \mathrm{H}, \mathrm{I}-\mathrm{NH})$, -1.59 br.s (1H, III-NH).

Chlorin $e_{6}$ 13-N,N-dimethylamide-15,17-dimethyl ester (10). Signals of the major atropoisomer: $9.75 \mathrm{~s}\left(1 \mathrm{H}, \mathrm{H}^{10}\right), 9.71 \mathrm{~s}$ $\left(1 \mathrm{H}, \mathrm{H}^{5}\right), 8.88 \mathrm{~s}\left(1 \mathrm{H}, \mathrm{H}^{20}\right), 8.16 \mathrm{dd}\left[1 \mathrm{H}, 3-\left(\mathrm{CH}=\mathrm{CH}_{2}\right), J 17.6\right.$ and $11.7 \mathrm{~Hz}], 6.41 \mathrm{~d}\left[1 \mathrm{H}, 3-\left(\mathrm{CH}=\mathrm{CH} H_{\text {trans }}\right), J 17.6 \mathrm{~Hz}\right], 6.18 \mathrm{~d}[1 \mathrm{H}$, $\left.3-\left(\mathrm{CH}=\mathrm{CH} H_{\mathrm{cis}}\right), J 11.7\right], 5.89 \mathrm{~d}\left(1 \mathrm{H}, \mathrm{H}^{15(1)}, J 19.1 \mathrm{~Hz}\right), 5.08$ $\mathrm{d}\left(1 \mathrm{H}, \mathrm{H}^{15(1)}{ }_{\mathrm{B}}, J \mathrm{~J} 19.1 \mathrm{~Hz}\right), 4.52 \mathrm{q}\left(1 \mathrm{H}, \mathrm{H}^{18}, J 6.6 \mathrm{~Hz}\right), 4.41 \mathrm{br} . \mathrm{d}$ $\left(1 \mathrm{H}, \mathrm{H}^{17}, J 9.5 \mathrm{~Hz}\right), 3.86 \mathrm{q}\left(2 \mathrm{H}, 8-\mathrm{CH}_{2} \mathrm{CH}_{3}, J 7.3 \mathrm{~Hz}\right), 3.85 \mathrm{~s}(3 \mathrm{H}$, $\left.15-\mathrm{CH}_{2} \mathrm{COOCH}_{3}\right), 3.68 \mathrm{~s}\left(3 \mathrm{H}, 17-\mathrm{CH}_{2} \mathrm{CH}_{2} \mathrm{COOCH}_{3}\right), 3.55 \mathrm{~s}(3 \mathrm{H}$, $\left.12-\mathrm{CH}_{3}\right), 3.54 \mathrm{~s}\left(3 \mathrm{H}, 2-\mathrm{CH}_{3}\right), 3.37 \mathrm{~s}\left(3 \mathrm{H}, 7-\mathrm{CH}_{3}\right), 13-\mathrm{CON}\left(\mathrm{CH}_{3}\right)_{2}$ : 3.50 and 2.78 (both s $3 \mathrm{H}) ; 2.66-208 \mathrm{~m}\left(4 \mathrm{H}, 17-\left(\mathrm{CH}_{2} \mathrm{CH}_{2} \mathrm{COOCH}_{3}\right)\right.$ ), $1.77 \mathrm{t}\left(3 \mathrm{H}, 8-\mathrm{CH}_{2} \mathrm{CH}_{3}, J 7.3 \mathrm{~Hz}\right), 1.76 \mathrm{~d}\left(3 \mathrm{H}, 18-\mathrm{CH}_{3}, J 6.6 \mathrm{~Hz}\right)$, -1.66 br.s. (1H, III-NH); -1.86 br.s $(1 \mathrm{H}, \mathrm{III}-\mathrm{NH})$. Signals of the minor atropoisomer: $9.73 \mathrm{~s}\left(1 \mathrm{H}, \mathrm{H}^{10}\right), 9.68 \mathrm{~s}\left(1 \mathrm{H}, \mathrm{H}^{5}\right), 8.83 \mathrm{~s}$ $\left(1 \mathrm{H}, \mathrm{H}^{20}\right), 8.14 \mathrm{dd}\left[1 \mathrm{H}, 3-\left(\mathrm{CH}=\mathrm{CH}_{2}\right), J 17.6\right.$ and $\left.11.7 \mathrm{~Hz}\right], 6.41$ d [1H, 3- $\left.\left(\mathrm{CH}=\mathrm{CH}_{\text {trans }}\right), J 17.6 \mathrm{~Hz}\right], 6.18 \mathrm{~d}\left[1 \mathrm{H}, 3-\left(\mathrm{CH}=\mathrm{CH} H_{\text {cis }}\right)\right.$, $J 11.7 \mathrm{~Hz}], 5.73 \mathrm{~d}\left(1 \mathrm{H}, \mathrm{H}^{15(1)}{ }_{\mathrm{A}}, J 19.1 \mathrm{~Hz}\right), 5.17 \mathrm{~d}\left(1 \mathrm{H}, \mathrm{H}^{15(1)}{ }_{\mathrm{B}}\right.$, $J 19.1 \mathrm{~Hz}), 4.52 \mathrm{q}\left(1 \mathrm{H}, \mathrm{H}^{18}, J 6.6 \mathrm{~Hz}\right), 4.46 \mathrm{br} . \mathrm{d}\left(1 \mathrm{H}, \mathrm{H}^{17}, J 8.1 \mathrm{~Hz}\right)$, $3.86 \mathrm{q}\left(2 \mathrm{H}, 8-\mathrm{CH}_{2} \mathrm{CH}_{3}, J 7.3 \mathrm{~Hz}\right), 3.80 \mathrm{~s}\left(3 \mathrm{H}, 15-\mathrm{CH}_{2} \mathrm{COOCH}_{3}\right)$, $3.65 \mathrm{~s}\left(3 \mathrm{H}, 17-\mathrm{CH}_{2} \mathrm{CH}_{2} \mathrm{COOCH}_{3}\right), 3.55 \mathrm{~s}\left(3 \mathrm{H}, 12-\mathrm{CH}_{3}\right), 3.53 \mathrm{~s}$ $\left(3 \mathrm{H}, 2-\mathrm{CH}_{3}\right), 3.37 \mathrm{~s}\left(3 \mathrm{H}, 7-\mathrm{CH}_{3}\right), 13-\mathrm{CON}\left(\mathrm{CH}_{3}\right)_{2}: 3.54$ and 3.14 (both s $3 \mathrm{H}) ; 2.66-208 \mathrm{~m}\left(4 \mathrm{H}, 17-\left(\mathrm{CH}_{2} \mathrm{CH}_{2} \mathrm{COOCH}_{3}\right)\right), 1.77 \mathrm{t}(3 \mathrm{H}$, $\left.8-\mathrm{CH}_{2} \mathrm{CH}_{3}, J 7.3 \mathrm{~Hz}\right), 1.69 \mathrm{~d}\left(3 \mathrm{H}, 18-\mathrm{CH}_{3}, J 6.6 \mathrm{~Hz}\right),-1.55$ br.s $(1 \mathrm{H}$, III-NH); -1.70 br.s (1H, III-NH).

Chlorin $e_{6}$ 13-N-methylamide-15,17-dimethyl ester (11): $9.73 \mathrm{~s}\left(1 \mathrm{H}, \mathrm{H}^{10}\right), 9.68 \mathrm{~s}\left(1 \mathrm{H}, \mathrm{H}^{5}\right), 8.84 \mathrm{~s}\left(1 \mathrm{H}, \mathrm{H}^{20}\right), 8.13 \mathrm{dd}[1 \mathrm{H}$, $3-\left(\mathrm{CH}=\mathrm{CH}_{2}\right), J 18.3$ and $\left.11.7 \mathrm{~Hz}\right], 6.39 \mathrm{q}\left(1 \mathrm{H}, 13-\mathrm{CONHCH}_{3}\right.$, $J 5.1 \mathrm{~Hz}), 6.40 \mathrm{~d}\left[1 \mathrm{H}, 3-\left(\mathrm{CH}=\mathrm{CH} H_{\text {trans }}\right), J 18.3 \mathrm{~Hz}\right], 6.18 \mathrm{~d}[1 \mathrm{H}$, $\left.3-\left(\mathrm{CH}=\mathrm{CH} H_{\text {cis }}\right), J 11.7 \mathrm{~Hz}\right], 5.56 \mathrm{~d}\left(1 \mathrm{H}, \mathrm{H}^{15(1)}{ }_{\mathrm{A}}, J 19.1 \mathrm{~Hz}\right), 5.29$ $\mathrm{d}\left(1 \mathrm{H}, \mathrm{H}^{15(1)}{ }_{\mathrm{B}}, J \mathrm{~J} 19.1 \mathrm{~Hz}\right), 4.50 \mathrm{q}\left(1 \mathrm{H}, \mathrm{H}^{18}, J^{\mathrm{A}} 7.3 \mathrm{~Hz}\right), 4.38$ br.d $\left(1 \mathrm{H}, \mathrm{H}^{17}, J 9.5 \mathrm{~Hz}\right), 3.86 \mathrm{~s}\left(3 \mathrm{H}, 15-\left(\mathrm{CH}_{2} \mathrm{COOCH}_{3}\right), 3.83 \mathrm{q}(2 \mathrm{H}\right.$,
$\left.8-\mathrm{CH}_{2} \mathrm{CH}_{3}, J 8.1 \mathrm{~Hz}\right), 3.65 \mathrm{~s}\left(3 \mathrm{H}, 17-\mathrm{CH}_{2} \mathrm{CH}_{2} \mathrm{COOCH}_{3}\right), 3.59 \mathrm{~s}$ $\left(3 \mathrm{H}, 12-\mathrm{CH}_{3}\right), 3.53 \mathrm{~s}\left(3 \mathrm{H}, 2-\mathrm{CH}_{3}\right), 3.35 \mathrm{~s}\left(3 \mathrm{H}, 7-\mathrm{CH}_{3}\right), 3.30 \mathrm{~d}$ $\left(3 \mathrm{H}, 13-\mathrm{CONHCH}_{3}, J 5.1 \mathrm{~Hz}\right), 17-\left(\mathrm{CH}_{2} \mathrm{CH}_{2} \mathrm{COOCH}_{3}\right): 2.65-$ $2.51 \mathrm{~m}(1 \mathrm{H}), 2.31-2.12 \mathrm{~m}(2 \mathrm{H})$ and $1.88-1.79 \mathrm{~m}(1 \mathrm{H}), 1.75 \mathrm{t}(3 \mathrm{H}$, $\left.8-\mathrm{CH}_{2} \mathrm{CH}_{3}, J 7.3 \mathrm{~Hz}\right), 1.75 \mathrm{~d}\left(3 \mathrm{H}, 18-\mathrm{CH}_{3}, J 7.3 \mathrm{~Hz}\right),-1.56$ br.s $(1 \mathrm{H}$, I-NH), -1.77 br.s (1H, III-NH).

\section{Results and Discussion}

This study describes the use of DOSY spectroscopy for the assessment of the influence of exchange processes on D coefficient values of inner N-H protons. The structures of the studied porphyrins were selected to present a variety of macrocycles and thus the chemical environment of $\mathrm{N}-\mathrm{H}$ protons. The structural details of these compounds result in significantly different chemical shifts and signal widths for $\mathrm{N}-\mathrm{H}$ protons. Among the test compounds there were porphyrins (1-4) and chlorins - derivatives of chlorophylls $a$ and $b$ with different peripheral substituents (5-11). The self-diffusion DOSY coefficients observed in deuterated chloroform for $\mathrm{C}-\mathrm{H}$ and $\mathrm{N}-\mathrm{H}$ protons are more or less different for all the tested porphyrins, due to the higher mobility of the latter (see Table 1). Figure 1 shows the spectrum of deuteroporphyrin dimethyl ester with clear differences of $\mathrm{D}$ values of $\mathrm{N}-\mathrm{H}$ protons from the values of the other protons in the molecule. The measured diffusion coefficients for C-H protons of the macrocyclic core and peripheral substituents more or less (in many cases considerably) differ from the corresponding coefficients for N-H protons, with the latter exhibiting greater values which demonstrates their higher mobility. The increase of the measured diffusion coefficient caused by exchange processes involving N-H protons, $\Delta \mathrm{D}_{\mathrm{ex}}$, can be denoted as follows:

$$
\Delta \mathrm{D}_{\mathrm{ex}}=\mathrm{D}_{\mathrm{NH}}-\mathrm{D}_{\mathrm{CH}},
$$

where $\mathrm{D}_{\mathrm{CH}}$ is the self-diffusion molecular coefficient found from the measurement of self-diffusion of all $\mathrm{C}-\mathrm{H}$ protons in the molecule, while $\mathrm{D}_{\mathrm{NH}}$ is the self-diffusion coefficient measured for $\mathrm{N}-\mathrm{H}$ protons. The increase of $\mathrm{D}$ for $\mathrm{N}-\mathrm{H}$

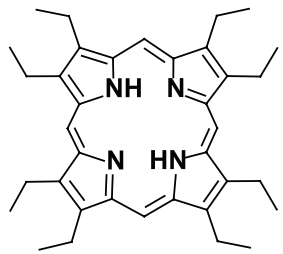

(1)

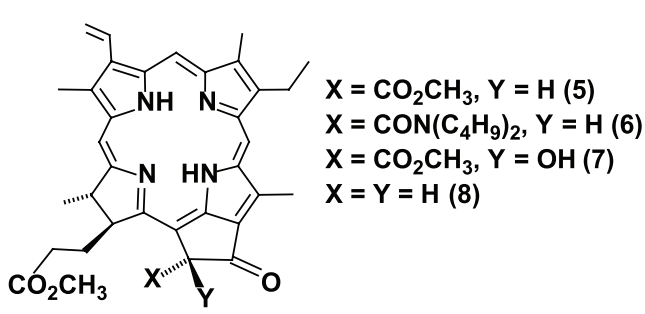

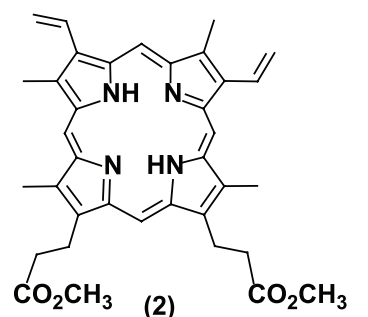

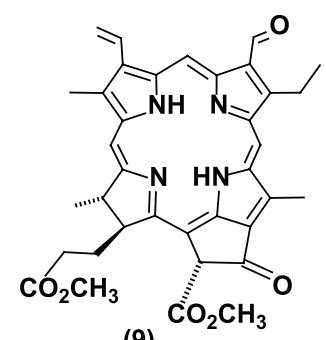

(9)
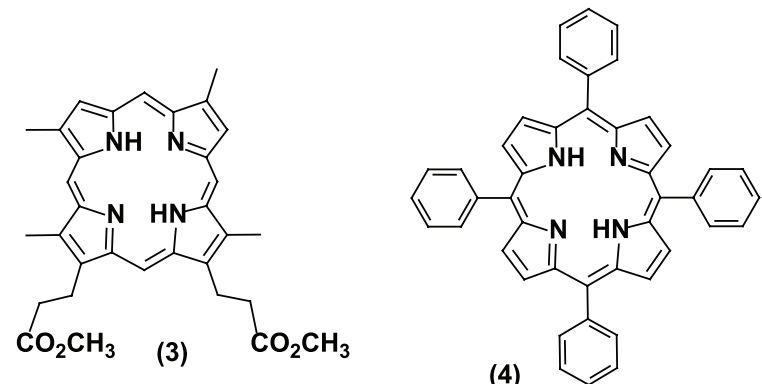

(4)

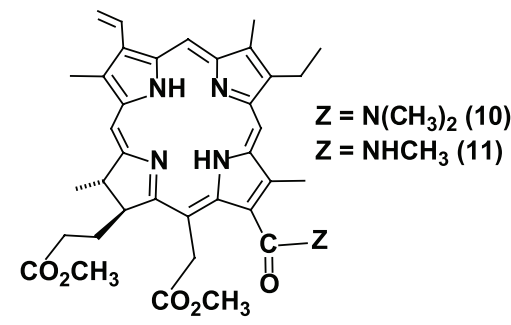

Scheme 1. 
protons can be explained by their involvement in intermolecular exchange processes (Scheme 2, reactions B) and by fast hydrogen exchange between nitrogen atoms in the $\mathrm{H}_{2} \mathrm{~N}_{4}$ coordination site ${ }^{[1-5]}$ (the so called N-NH-tautomerism, Scheme 2, reactions A). The contribution of the intramolecular exchange to the $\mathrm{D}$ values for $\mathrm{N}-\mathrm{H}$ is low due to the fact that during the diffusion time (D20=0.1 s) an N-H proton can move from its initial position no further than $4 \AA .^{[11]}$ This corresponds to a diffusion coefficient of about $10^{-13} \mathrm{~cm}^{2} / \mathrm{s}$, three orders of magnitude less than DOSY lower detection limit $\left(10^{-10} \mathrm{~cm}^{2} / \mathrm{s}\right)$. The increase of the measured $\mathrm{D}$ coefficient for $\mathrm{N}-\mathrm{H}$ protons can be in this case caused by intermolecular proton exchange between porphyrins and residual water (its concentration of the deuterated solvent is almost the same as the concentration of the test porphyrins). Intermolecular exchange effects in DOSY have been described ${ }^{[12-15]}$ and manifest in the same manner as in our experiments. The measured D coefficient for protons involved in exchange processes with water molecules are greater than those for the protons not involved in exchange. This is because the protons participating in the exchange are able to diffuse as a part of more mobile water molecule for some time. In order to receive further confirmation of the intermolecular nature of exchange processes we measured D for N-H protons of compound (11) under the condition of a greater water content (the concentration of water increased 7-fold, up to $0.07 \mathrm{mmol} / \mathrm{mL}$ ). The value of $\Delta \mathrm{D}_{\mathrm{ex}}$ then increased for both $\mathrm{N}-\mathrm{H}$ protons (up to $1.35 \cdot 10^{-9}$ and $4.86 \cdot 10^{-9} \mathrm{~m}^{2} / \mathrm{s}$ respectively). Clearly, the higher the difference of self-diffusion coefficients of N-H protons and other protons of the porphyrinic molecule, the more intensive the exchange processes involving these inner protons.

The value of $\Delta \mathrm{D}_{\mathrm{ex}}$ is structure-dependent and can act as a quantitative measure of exchange intensity. Obviously, when the activation energies of the tautomeric reactions are comparable, $\Delta \mathrm{D}_{\mathrm{ex}}$ can indicate the acidity of $\mathrm{N}-\mathrm{H}$ bond and its polarity. The absence of harsh conditions during the test procedure allows to extend the application sphere for this quantitative parameter. The analysis of the impact of structure on $\Delta \mathrm{D}_{\mathrm{ex}}$ values reveals a number of interesting correlations. In the case of compounds (1-4) with a porphyrinic chromophore the $\mathrm{N}-\mathrm{H}$ protons are equivalent, their mobility is the same and $\Delta \mathrm{D}_{\mathrm{ex}}$ value is comparable to the values for the non-equivalent macrocyclic protons in 13-amide derivatives of chlorin $e_{6}$. The difference between the diffusion coefficients of $\mathrm{N}-\mathrm{H}$ protons and the protons of the whole molecule can be a more reliable information source on the polarity of N-H bond than the chemical shift of N-H, as the latter depends on the structure of the aromatic system and the strength of the ring current in particular. So, DOSY experiment data demonstrate that $\mathrm{N}-\mathrm{H}$ bond polarities are comparable in porphyrins and chlorins without an exo-cycle, though the corresponding chemical shifts differ considerably. Furthermore, meso-substituted porphyrinic core (compound (4)) exhibited less intensive exchange processes compared to porphyrins with free meso positions (1-3) (in the case of TPP, $\Delta \mathrm{D}_{\text {ex }}$ is about $0.3 \cdot 10^{-9} \mathrm{~m}^{2} / \mathrm{s}$, in contrast to $0.7-1.9 \cdot 10^{-9}$ $\mathrm{m}^{2} / \mathrm{s}$ for $\left.(\mathbf{1 - 3})\right)$.

Noteworthy, replacement of the methyl group in position 7 of methyl pheophorbide $a$ with a formyl group (thus converting methyl pheophorbide $a$ (5) into methyl pheophorbide $b(9))$ results in a complete loss of mobility of $\mathrm{NH}$ protons, though an increase of exchange is expected as an electron-withdrawing group is introduced what should result in polarization of $\mathrm{N}-\mathrm{H}$ bond and facilitate its heterolysis. The experimental data can be explained by a decrease of the basicity of the inner $\mathrm{N}$ atoms due to the introduction of the electron-withdrawing formyl group.

It appeared that for $\mathrm{NH}$ protons that belong to the same macrocycle, the stronger the field, the higher the proton mobility. Considering the shielding provided by the ring currents almost equal, the chemical shift differences are

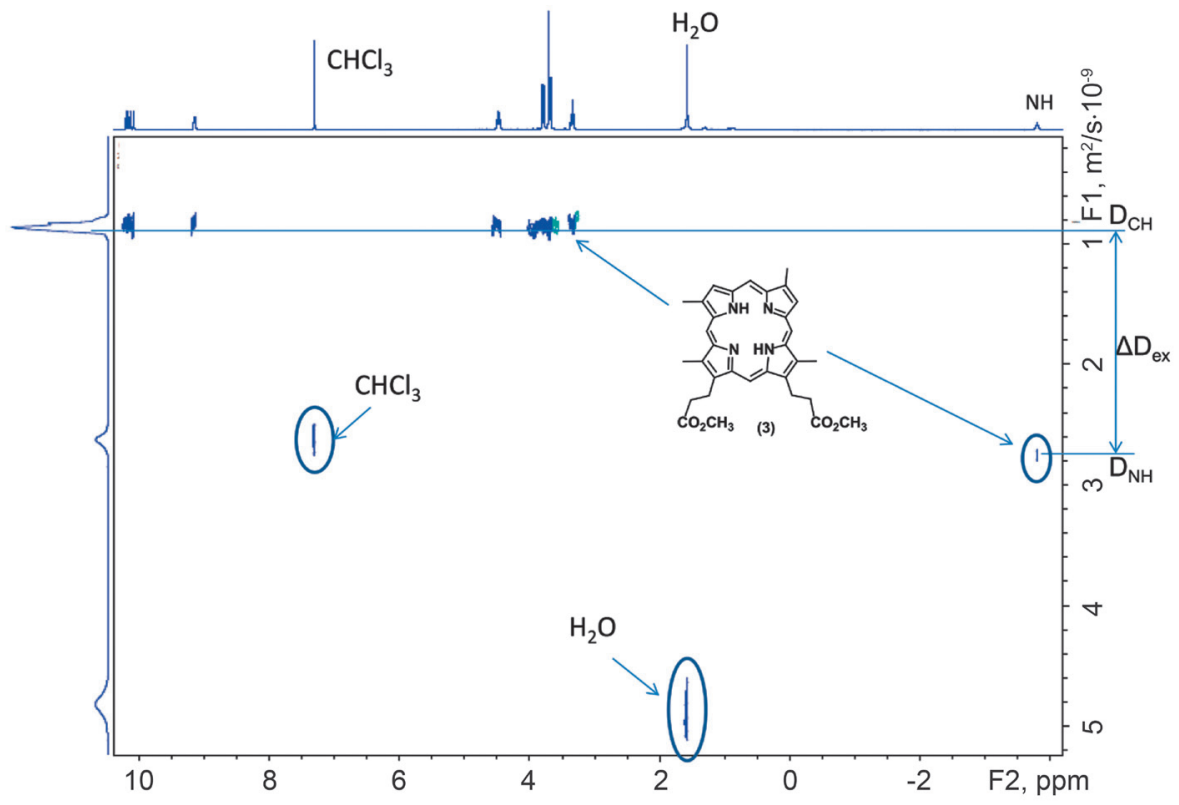

Figure 1. DOSY spectra of deuteroporphyrin dimethyl ester (3) $\left(\mathrm{CDCl}_{3}, 300 \mathrm{MHz}\right)$. 
Table 1. DOSY experimental data for porphyrins (1-11): measured self-diffusion coefficients of the whole molecule (for $\mathrm{C}-\mathrm{H}$ protons) $\left(D_{\mathrm{CH}}\right)$ and for $\mathrm{NH}$ protons $\left(\mathrm{D}_{\mathrm{NH}}\right)$, the instrumental error does not exceed $10 \%$.

\begin{tabular}{cccc}
\hline Compound & $\begin{array}{c}\mathrm{D}_{\mathrm{CH}}\left(\cdot 10^{9}\right) \\
\mathrm{m}^{2} / \mathrm{s}\end{array}$ & $\begin{array}{c}\mathrm{D}_{\mathrm{NH}}\left(\cdot 10^{9}\right) \mathrm{m}^{2} / \mathrm{s} \\
\left(\delta_{\mathrm{NH}}, \mathrm{ppm}\right)\end{array}$ & $\begin{array}{c}\Delta \mathrm{D}_{\mathrm{ex}}\left(\cdot 10^{9}\right) \mathrm{m}^{2} / \mathrm{s} \\
\left(\delta_{\mathrm{NH}}, \mathrm{ppm}\right)\end{array}$ \\
\hline $\mathbf{1})$ & 0.85 & $2.28(-3.70)$ & 1.43 \\
$(\mathbf{2})$ & 0.76 & $1.70(-3.87)$ & 0.94 \\
$(\mathbf{3})$ & 0.87 & $2.76(-3.80)$ & 1.89 \\
$(\mathbf{4})$ & 0.77 & $1.09(-2.72)$ & 0.32 \\
$(\mathbf{5})$ & 0.77 & $0.81(-1.58)$ & $0.04(-1.58)(\mathrm{I}-\mathrm{NH})$ \\
& & $2.34(0.59)$ & $1.57(0.59)(\mathrm{III}-\mathrm{NH})$ \\
$(\mathbf{6})$ & 0.75 & $0.76(-1.64)$ & $0.00(-1.64)(\mathrm{I}-\mathrm{NH})$ \\
& & $5.44(0.46)$ & $4.69(0.46)(\mathrm{III}-\mathrm{NH})$ \\
$(\mathbf{7})$ & 0.74 & $1.06(-1.861)$ & $0.32(-1.86)(\mathrm{I}-\mathrm{NH})$ \\
& & $4.34(0.364)$ & $3.60(0.36)(\mathrm{III}-\mathrm{NH})$ \\
$(\mathbf{8})$ & 0.78 & $0.90(-1.64)$ & $0.12(-1.64)(\mathrm{I}-\mathrm{NH})$ \\
& & $5.44(0.52)$ & $4.66(0.52)(\mathrm{III}-\mathrm{NH})$ \\
$(\mathbf{9})$ & 0.74 & $0.74(-1.59)$ & $0.00(-1.59)(\mathrm{I}-\mathrm{NH})$ \\
& & $0.74(0.49)$ & $0.00(0.49)(\mathrm{III}-\mathrm{NH})$ \\
$(\mathbf{1 0})$ & 0.76 & $0.92(-1.86)$ & $0.16(-1.86)(\mathrm{I}-\mathrm{NH})$ \\
& & $2.13(-1.66)$ & $1.37(-1.66)(\mathrm{III}-\mathrm{NH})$ \\
& 0.76 & $1.11(-1.70)$ & $0.35(-1.70)(\mathrm{I}-\mathrm{NH})$ \\
& & $3.79(-1.55)$ & $3.03(-1.55)(\mathrm{III}-\mathrm{NH})$ \\
$(\mathbf{1 1})$ & 0.75 & $1.01(-1.77)$ & $0.26(-1.77)(\mathrm{I}-\mathrm{NH})$ \\
& & $2.40(-1.56)$ & $1.65(-1.56)(\mathrm{III}-\mathrm{NH})$ \\
\hline
\end{tabular}

caused by the difference in N-H bond polarity: the more polar the bond is, the weaker field the signal for this proton is in. On one hand, a lower bond polarity corresponds to a greater electronic density on the proton providing better shielding. On the other hand, more polar bonds undergo heterolysis easier, which results in a more pronounced involvement of the proton in exchange processes. It is worthy of notice that inner N-H proton of pyrrolic ring I is comparably weakly involved in exchange processes. Signals of more mobile inner $\mathrm{N}-\mathrm{H}$ protons not only lay downfield compared to the less mobile ones, but also are broadened. This allows to conclude that signal broadening for $\mathrm{N}-\mathrm{H}$ protons is to a great extent caused by their intermolecular exchange and the contribution of quadrupole effect is insignificant. For example, ${ }^{1} \mathrm{H}$ NMR signal broadening for the $\mathrm{N}-\mathrm{H}$ proton in pyrrolic ring III of the tested phorbine derivatives corresponds to more intensive hydrogen exchange than for I-NH proton. This is also true for the non-equivalent inner $\mathrm{N}-\mathrm{H}$ protons in chlorine derivatives (10) and (11).

It is noteworthy that regardless of deuteroporphyrin (3) being non-symmetric, its inner N-H protons are magnetically equivalent and are equally involved in exchange processes. This leads to the conclusion that not only non-symmetrical substitution is required to provide a difference in $\mathrm{N}-\mathrm{H}$ chemical shifts and the degree of participation in hydrogen exchange, but lowering of symmetry, as in chlorins, is also needed. The presence of an exo-cycle augments the effect of the macrocycle symmetry lowering both for the
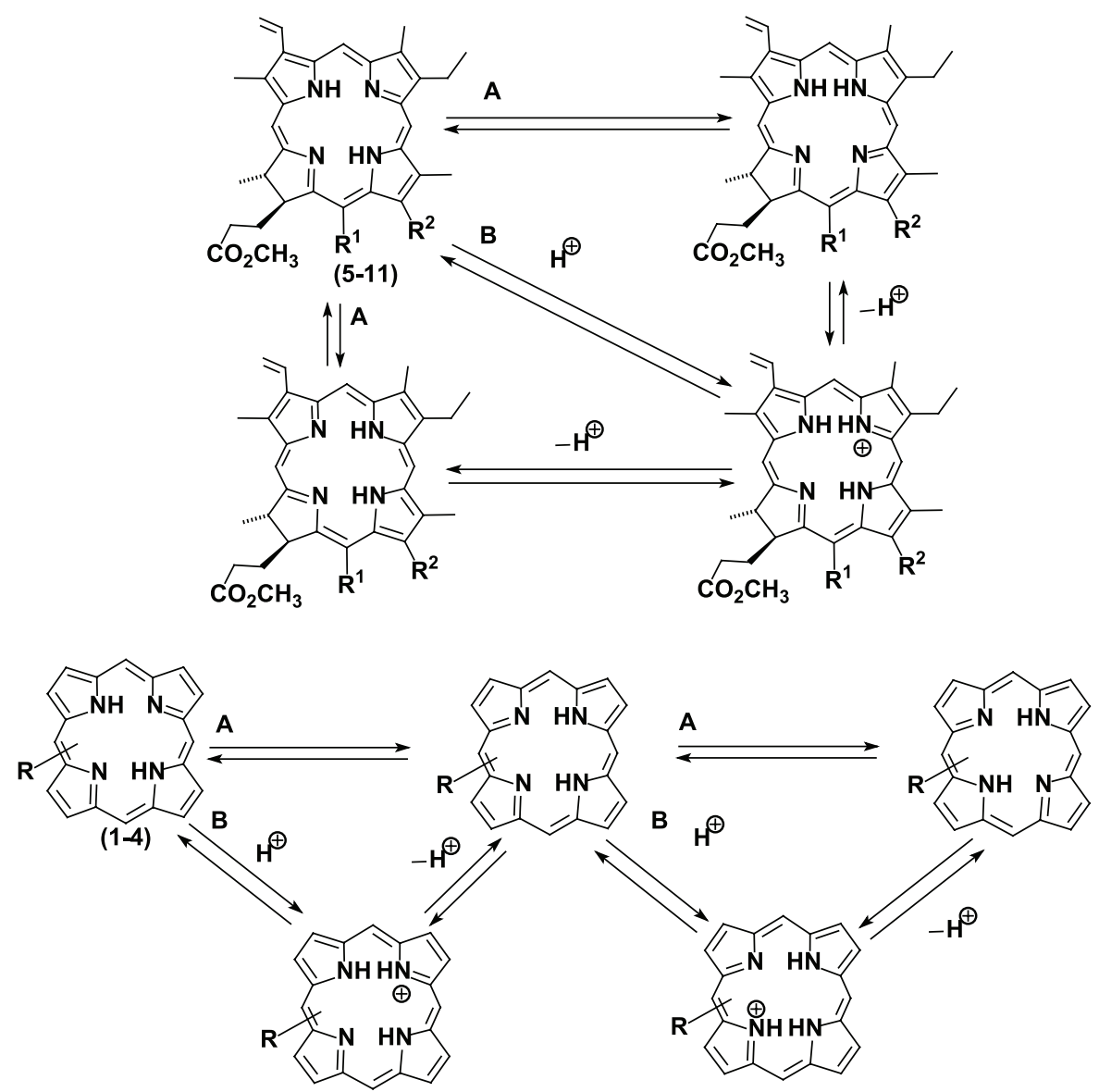

Scheme 2. 
chemical shifts of inner N-H protons and their mobility with respect to exchange processes. Weaker structural factors can also contribute: replacement of a primary amide group in position 13 with a secondary one results in a significant change in $\Delta \mathrm{D}_{\mathrm{ex}}$; furthermore, this value considerably differs for atropoisomers in the case of a tertiary 13-dimethyl amide (10).

\section{Conclusion}

Summing up, the current study demonstrates the involvement of the inner N-H protons of porhyrinic compounds in exchange processes can be determined by DOSY NMR spectroscopy as the difference between the observed diffusion coefficients of these protons and $\mathrm{C}-\mathrm{H}$ protons of the rest of the molecule. The measured diffusion coefficients of $\mathrm{C}-\mathrm{H}$ protons are less significant. The difference of the measured N-H and C-H diffusion coefficients can be a qualitative measure for the intensity of intermolecular exchange processes and as a result - of N-H acidity. The absence of harsh experimental conditions extends the applicability range of the proposed criterion. Using DOSY spectroscopy allows to obtain new information about the state of $\mathrm{N}-\mathrm{H}$ protons in porphyrinic macrocycles. Our experimental data show that regardless of a great variety in chemical shifts, the N-H acidity in porhyrins and chlorins are similar. Furthermore, we established that non-equivalent $\mathrm{N}-\mathrm{H}$ protons in chlorins are unequally involved in exchange processes.

\section{References}

1. Berezin D.B. Macrocyclic Effect and Structural Chemistry of Porphyrins. Moscow: Krasand, 2010. 424 p. (in Russ.)
[Березин Д.Б. Макрочиклический эффект и структурная химия порфиринов. М.: Красанд, 2010. 421 с.].

2. Berezin B.D. Coordination Compounds of Porphyrins and Phthalocyanine. Moscow: Nauka, 1978. 280 p. (in Russ.) [Березин Б.Д. Координационные соединения порфиринов и фталоцианина. М.: Наука, 1978. 280 с.].

3. Smith K.M. Porphyrins and Metalloporphyrins. Amsterdam: Elsevier, 1975. $890 \mathrm{p}$.

4. Berezin B.D., Berezin D.B. Chromophore Systems of Macrocycles and Linear Molecules. Moscow: Krasand, 2013. 240 p. (in Russ.) [Березин Б.Д., Березин Д.Б. Хромофорные системы макрочиклов и линейных молекул. М.: Красанд, 2014. 240 c.].

5. Gurinovich G.P., Sevchenko A.N., Solovyov K.N. Spectroscopy of Chlorophyll and Related Compounds, Minsk: Nauka i Tekhnika, 1968. 520 p. (in Russ.) [Гуринович Г.П., Севченко А.Н., Соловьев К.Н. Спектроскопия хлорофилла и родственных соединений. Минск: Наука и техника, 1968. 520 c.].

6. Porphyrins: Structure, Properties, Synthesis (Enikolopyan N.S., Ed.). Moscow: Nauka, 1985, 334 p. (in Russ.) [Порфиринь:: структура, свойства, синтез (Ениколопян Н.С., ред.). М.: Наука, 1985. 333 с.].

7. Kuchin A.V., Karmanova L.P., Belykh D.V. Method for obtaining of methyl pheophorbide $a$ ester. Patent RF № 2180342.

8. Belykh D.V., Kopylov E.A., Gruzdev I.V., Kuchin A.V. Russ. J. Org. Chem. 2010, 46, 577-585.

9. Belykh D.V., Karmanova L.P., Kuchin A.V., Spirikhin L.V. Russ. J. Org. Chem. 2007, 43, 126-134.

10. Ellsworth P.A., Storm C.B. J. Org. Chem. 1978, 43, 281-283.

11. Lauher J.W., Ibers J.A. J. Am. Chem. Soc. 1973, 95, 51485152 .

12. Thureau P., Ancian B., Viel S., Thevand A. Chem. Commun. 2006, 200-202.

13. Cabrita E.J., Berger S. Magn. Reson. Chem. 2002, 40, 122127.

14. Cabrita E.J., Berger S., Bräuer P., Kärger J. J. Magn. Res. 2002, 157, 124-131.

15. Dolores Diaz M., Berger S. Carbohydr. Res. 2000, 329, 1-5.

Received 16.05.2016

Revised 15.01.2017

Accepted 25.03.2017 